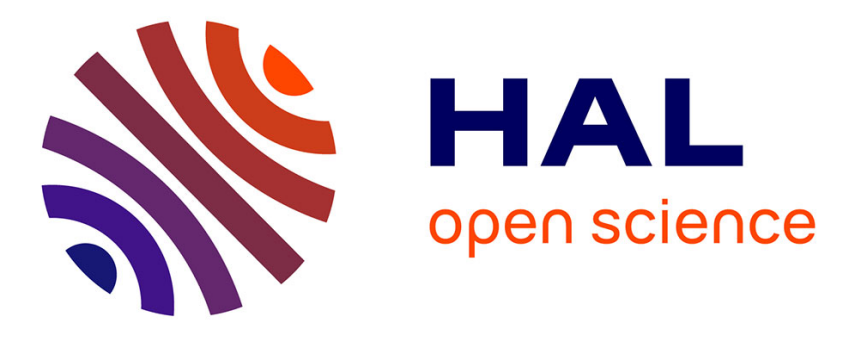

\title{
Passive Models of Viscothermal Wave Propagation in Acoustic Tubes
}

\author{
Stefan Bilbao, Reginald Harrison, Jean Kergomard, Bruno Lombard, \\ Christophe Vergez
}

\section{- To cite this version:}

Stefan Bilbao, Reginald Harrison, Jean Kergomard, Bruno Lombard, Christophe Vergez. Passive Models of Viscothermal Wave Propagation in Acoustic Tubes. Journal of the Acoustical Society of America, 2015, 138, pp.555-558. 10.1121/1.4926407 . hal-01151253v2

\section{HAL Id: hal-01151253 \\ https://hal.science/hal-01151253v2}

Submitted on 28 Apr 2016

HAL is a multi-disciplinary open access archive for the deposit and dissemination of scientific research documents, whether they are published or not. The documents may come from teaching and research institutions in France or abroad, or from public or private research centers.
L'archive ouverte pluridisciplinaire HAL, est destinée au dépôt et à la diffusion de documents scientifiques de niveau recherche, publiés ou non, émanant des établissements d'enseignement et de recherche français ou étrangers, des laboratoires publics ou privés. 


\title{
Passive Models of Viscothermal Wave Propagation in Acoustic Tubes
}

\author{
Stefan Bilbao ${ }^{\text {a) }}$ and Reginald Harrison \\ Acoustics and Audio Group, University of Edinburgh, Kings Buildings, Mayfield Rd., Edinburgh, EH9 3JZ, \\ United Kingdom \\ Jean Kergomard, Bruno Lombard, and Christophe Vergez \\ Laboratoire de Mécanique et Acoustique, CNRS-UPR 7051, Université Aix-Marseille, Centrale Marseille, \\ 13402 Marseille Cedex 20, France
}

(Dated: June 10, 2015)

\begin{abstract}
A continued fraction expansion to the immittances defining viscothermal wave propagation in a cylindrical tube has been presented recently in this journal, intended as a starting point for time domain numerical method design. Though the approximation has the great benefit of passivity, or positive realness under truncation, its convergence is slow leading to approximations of high order in practice. Other passive structures, when combined with optimisation methods, can lead to good accuracy over a wide frequency range, and for relatively low order.
\end{abstract}

PACS numbers: 43.20.Mv, 43.20.Hq, 43.55.Ev, 43.75.Fg

\section{INTRODUCTION}

The authors of a recent $\operatorname{article}^{1}$ propose a novel approximation to viscothermal losses in a cylindrical tube based on continued fraction expansions, in frequency, of exact expressions for impedance and admittance. The idea is to show that such an approximation is a suitable starting point for time domain numerical methods. In particular, the method proposed serves to sidestep difficulties encountered when high-frequency approximations are employed - while useful in the frequency domain, these lead to fractional derivatives in the resulting time domain system, and must be further approximated to lead to a recursive method.

The approach offers many advantages - and most importantly structural passivity, due to the non-negativity of element values in the resulting circuit representations, which are ladder structures of Cauer type, resulting from a continued fraction expansion. In this representation, the immittances are positive real functions, and remain so under truncation to any order. Not all such representations have this property - see, e.g., the article by Kergomard $^{2}$. Such structures are thus excellent candidates for the construction of time domain schemes ${ }^{3}$. The authors provide some numerical results in the form of fractional error in the real part of acoustic tube immittances as a function of frequency, for various orders of truncation of the approximation. As expected, such approximants perform very well in the low frequency range (as the continued fraction expansion is about the DC frequency), but converge slowly as the approximation order is increased; this order must be chosen high if one is interested in performing a simulation over a large range of frequencies.

The aim of this short article is to show other passive representations for viscothermal wave propagation which

\footnotetext{
a) Electronic address: sbilbao@staffmail.ed.ac.uk
}

are amenable to optimisation and operation over a wide frequency range, even at low order. Here, the point of view taken is broadly similar to that of Thompson: from a given immittance function, derive a low order rational and positive real approximant, which is suitable for use in a time domain numerical method. Instead of making use of a continued fraction expansion, however, a different structure is proposed, namely that of Foster. The element values defining such a structure could, in principle, be derived through a partial fraction expansion of the immittance. Here, however, the structure is used as a starting point for optimization procedures, leading ultimately to structures which perform well over a large range of frequencies even at low order, while sacrificing some accuracy in the low frequency range.

Supplementary material is available at

http://www.ness-music.eu/working-papers

\section{IMMITTANCES FOR A CYLINDRICAL TUBE}

A standard model of wave propagation in a cylindrical tube can be written in the frequency domain in terms of a pair of equations relating the acoustic pressure $P=$ $P(\omega, x)$ and acoustic volume velocity $U=U(\omega, x)$ :

$$
\partial_{x} P+Z U=0 \quad \partial_{x} U+Y P=0
$$

See. e.g., the article by Keefe ${ }^{4}$ and the references therein. Here, $x \in[0, L]$ is a spatial coordinate, for some tube length $L$, and $\omega$ is a frequency variable, in radians/s. $Z=Z(j \omega)$ and $Y=Y(j \omega)$ are the series impedance and shunt admittance respectively for the tube. Expressions for $Z$ and $Y$ are given as follows:

$$
Z=\frac{j \omega \rho}{\pi a^{2}} \frac{1}{1-F_{v}} \quad Y=\frac{j \omega \pi a^{2}}{\rho c^{2}}\left(1+(\gamma-1) F_{t}\right)
$$

where here, $\rho$ is air density, in $\mathrm{kg} / \mathrm{m}^{3}, c$ is the wave speed in $\mathrm{m} / \mathrm{s}, \gamma$ is the ideal gas constant and $a$ is the tube radius 
in $\mathrm{m}$. The functions $F_{v}$ and $F_{t}$ are defined by

$$
F_{v}=\phi\left(\sqrt{-j} r_{v}\right) \quad F_{t}=\phi\left(\sqrt{-j} r_{t}\right) \quad \phi(\zeta)=\frac{2 J_{1}(\zeta)}{\zeta J_{0}(\zeta)}
$$

where $j=\sqrt{-1}, J_{0}$ and $J_{1}$ are zeroth and first order Bessel functions and $r_{v}$ and $r_{t}$ are defined as

$$
r_{v}=a \sqrt{\rho \omega / \eta} \quad r_{t}=a \nu \sqrt{\rho \omega / \eta}
$$

with viscosity coefficient $\eta$ and Prandtl number $\nu^{2}=$ $C_{p} \eta / \kappa$, written in terms of the specific heat at constant pressure and $\kappa$ is thermal conductivity.

The immittances $Z$ and $Y$ may be split as

$$
Z=Z_{l}+Z_{v} \quad Y=Y_{l}+Y_{t}
$$

where $Z_{l}=j \omega \rho / \pi a^{2}$ and $Y_{l}=j \omega \pi a^{2} / \rho c^{2}$ are the immittances corresponding to wave propagation in the absence of viscous (respectively thermal) losses, and where

$$
Z_{v}=\frac{j \omega \rho}{\pi a^{2}} \frac{F_{v}}{1-F_{v}} \quad Y_{t}=\frac{j \omega \pi a^{2}}{\rho c^{2}}(\gamma-1) F_{t}
$$

represent the additional contributions due to such effects.

When extended to the entire complex plane, through the variable $s$, where $\operatorname{Im}(s)=\omega$, it is useful to examine the pole/zero structure of $Z_{v}(s)$ and $Y_{t}(s)$. See Figure 1. Both $Z_{v}$ and $Y_{t}$ possess interlaced poles and zeros on the negative real axis with a zero closest to the origin in both cases. This arrangement suggests particular circuit representations, as will be described shortly.
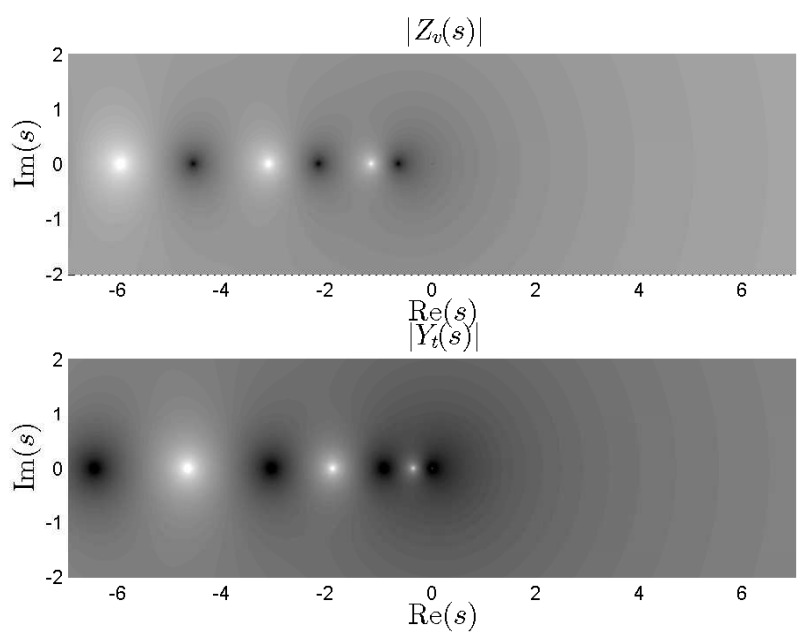

FIG. 1. Impedance magnitude $|Z(s)|$ (top) and admittance magnitude $|Y(s)|$ (bottom), plotted in the complex plane, in dB. Dark and light spots represent zeros and poles, respectively.

\section{CIRCUIT REPRESENTATIONS}

The breakdown of the immittances $Z$ and $Y$ into lossless and viscothermal effects as per (5) may be represented simply through a circuit diagram, using the analogy between pressure with voltage, and volume velocity
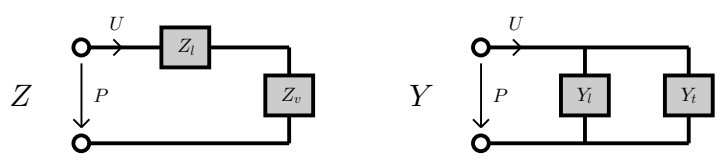

FIG. 2. Circuit representation of the breakdown of the impedance $Z=Z_{l}+Z_{v}$ and admittance $Y=Y_{l}+Y_{t}$.

with current, leading to a series combination for $Z$ and a parallel combination for $Y$. See Figure 2 .

Given the observation that the immittances $Z_{v}$ and $Y_{t}$ possess poles and zeros on the negative real axis, it is natural to seek approximations which are of two-element type ${ }^{5}-R L$ in the case of $Z_{v}$, and $R C$ in the case of $Y_{t}$. Furthermore, the approximations should be positive real and thus passive - a condition which is simply met if the network components take on non-negative coefficient values. A continued fraction expansion of the immittances about DC leads to ladder structures sometimes referred to as Cauer's second form ${ }^{5}$, as employed recently in the article by Thompson et $\mathrm{al}^{1}$. See Figure 3 .

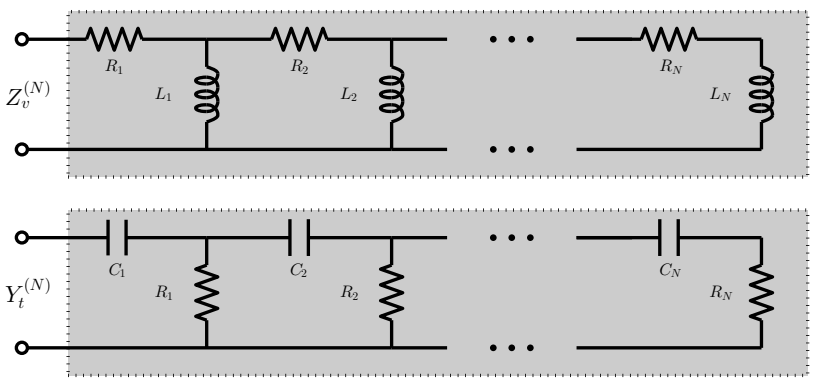

FIG. 3. Cauer structures corresponding to approximations $Z_{v}^{(N)}$ to $Z_{v}$ (top) and $Y_{t}^{(N)}$ to $Y_{t}$ (bottom), employing $N$ branches, with element values $R_{j}$ and $L_{j}, j=1, \ldots, N$ for the impedance $Z_{v}^{(N)}$, and $R_{j}$ and $C_{j}, j=1, \ldots, N$ for the admittance $Y_{t}^{(N)}$.

In practice, the ladder is truncated after a finite number $N$ of branches, leading to an approximation $Z_{v}^{(N)}$. As expected, the approximation is excellent in the low frequency range, and increases in accuracy with $N$. See Figure 4, to be compared with Figures 4 and 5 from Thompson et al. ${ }^{1}$. Here, and henceforth, the tube is assumed to be of radius $7.5 \mathrm{~mm}$, and the various constants are set to values corresponding to a temperature of $26.85^{\circ} \mathrm{C}$, using formulae given by Keefe ${ }^{4}$. Notice in particular that the approximation converges slowly - for this tube, a fourth order approximation produces results of reasonable accuracy up to about $20 \mathrm{~Hz}$. For the study of wind instruments, approximation to at least order 16 , giving reliable results up to the mid $\mathrm{kHz}$ range, would be necessary. 

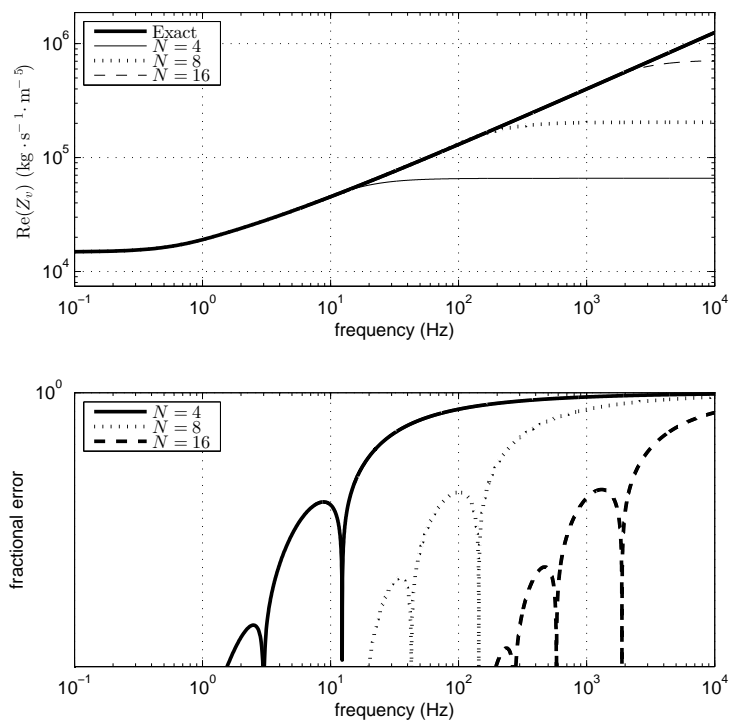

FIG. 4. Top: Real part of the impedance $Z_{v}$, and of continued fraction approximations $Z_{v}^{(N)}$, to various orders $N$, as indicated. Bottom: fractional error, as a function of frequency.

\section{A. Optimisation and Numerical Results}

Another approach, perhaps better suited to efficient numerical implementation, is optimisation of an approximation to the impedance ${ }^{6}$. The Cauer structure has the great benefit of structural passivity, due to the nonnegativity of all the element values. But other structures are also available - examples are the two element $R L$ and $R C$ Foster networks, shown in Figure 5.

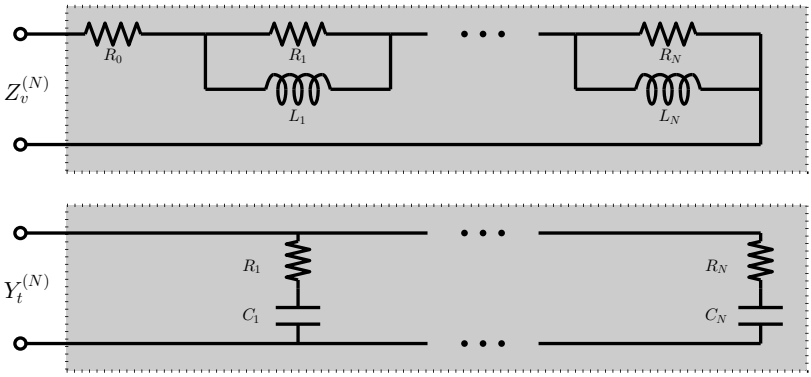

FIG. 5. Foster structures corresponding to approximations $Z_{v}^{(N)}$ to $Z_{v}$ (top) and $Y_{t}^{(N)}$ to $Y_{t}$ (bottom), employing $N$ branches, with element values $R_{j}$ and $L_{j}, j=1, \ldots, N$ for the impedance $Z_{v}^{(N)}$, and $R_{j}$ and $C_{j}, j=1, \ldots, N$ for the admittance $Y_{t}^{(N)}$. Note the additional resistance $R_{0}$ in the case of the Foster form for the impedance $Z_{v}$, allowing for a non-zero limiting low-frequency impedance.

The immittances $Z_{v}^{(N)}$ and $Y_{t}^{(N)}$ may be decomposed as sums,

$$
\begin{aligned}
Z_{v}^{(N)}(j \omega) & =R_{0}+\sum_{p=1}^{N} \frac{R_{p} L_{p} j \omega}{R_{p}+L_{p} j \omega} \\
Y_{t}^{(N)}(j \omega) & =\sum_{p=1}^{N} \frac{C_{p} j \omega}{1+R_{p} C_{p} j \omega}
\end{aligned}
$$

allowing for easy evaluation of derivatives with respect to the free parameters $R_{0}$ and $R_{q}, L_{q}, q=1, \ldots, N$ in the case of $Z_{v}^{(N)}$, or $R_{q}, C_{q}, q=1, \ldots, N$ in the case of $Y_{t}^{(N)}$. $\left(R_{0}\right.$ may be left free, or constrained to take on the limiting value $Z_{v}(0)=8 \eta / \pi a^{4}$.) In what follows, only the series impedance $Z_{v}$ will be discussed - the case of the shunt admittance $Y_{t}$ is similar.

At this point, a wide variety of choices are available to the designer regarding the range of frequencies over which to optimise, the cost function and the method of optimisation - these should clearly be chosen according to the application at hand. In the interest of reducing computational complexity in resulting time domain numerical designs, it is perhaps useful to examine the behaviour of the approximation over a wide range of frequencies, and employing a cost function $E$, dependent on the circuit parameters:

$$
E=\sum_{m=1}^{M}\left|1-Z_{v}^{(N)}\left(j \omega_{m}\right) / Z_{v}\left(j \omega_{m}\right)\right|^{2}
$$

for a set of $M$ exponentially spaced frequencies $\omega_{m}, m=$ $1 \ldots, M$. It is straightforward to include a weighting in the above cost function, so as to provide better accuracy over a desired frequency range.

Some results are shown in Figure 6 , for a tube of radius $7.5 \mathrm{~mm}$, and optimised over the range of frequencies from $0.1 \mathrm{~Hz}$ to $10000 \mathrm{~Hz}$. A gradient descent technique was used, with $M=100$ frequency values, and circuit values constrained to be positive. For $N=4$, for example, the optimised structure gives a reduced error relative to the continued fraction expansion from approximately $20 \mathrm{~Hz}$ upwards. For $N=16$, the optimised structure has an error on the order of 0.01 per cent over the entire range of frequencies. Outside the high-frequency limit of the optimisation range, the fit naturally becomes poorer, but remains superior to the continued fraction expansion.

\section{CONCLUDING REMARKS}

The article by Thompson et al. is clearly geared towards designing time domain methods to be used for the simulation of viscothermal effects in tubes - and as such, the passive Cauer structure arrived at through continued fraction expansion is an extremely useful one, as it allows for a stable numerical design. The main powerful insight is that it is possible to begin from the exact expression for the losses, rather than a derived form, often leading to fractional derivatives in the resulting time domain approximation $^{7}$. In short, it can be simpler to work with the exact expression, which is clearly advantageous. 

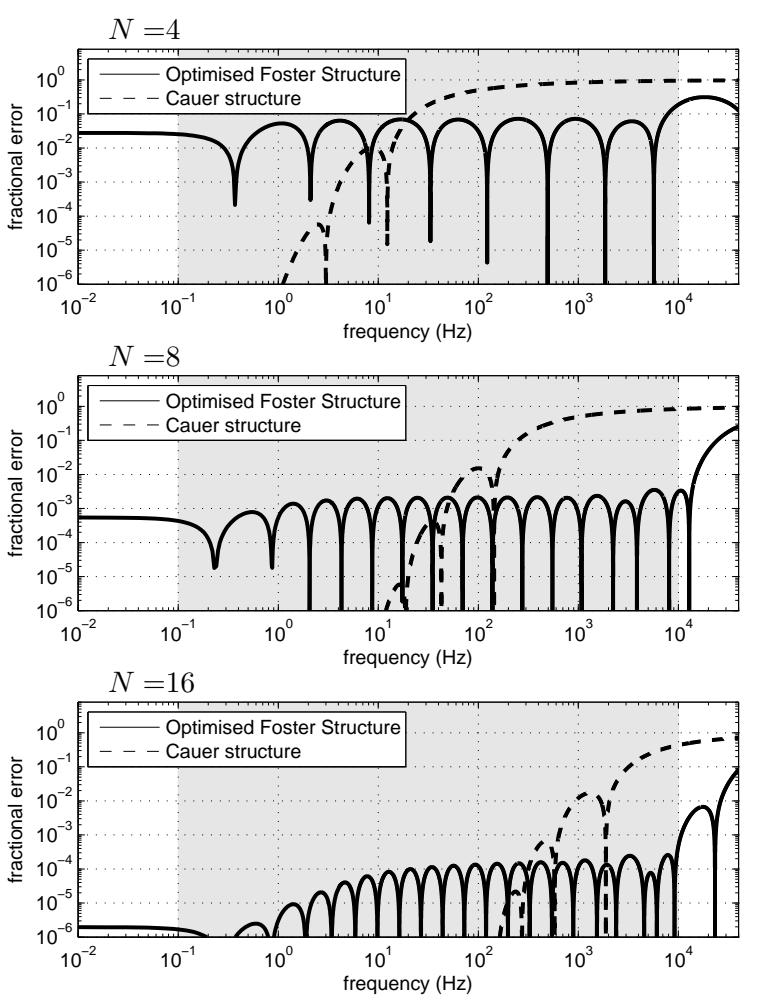

FIG. 6. Fractional error in the real part of the impedance $Z_{v}$ against frequency for Cauer structures and optimised Foster structures, for approximations of order $N=4,8$ and 16 . The range of frequencies over which the Foster structure is optimised is indicated by the shaded gray region.

Yet, if a time domain numerical method is the eventual goal, then it must be borne in mind that the order of the approximation to the loss terms will dominate calculation time and memory costs. The response of the Cauer structure matches that of the model very well in the low frequency range, and in some applications, this is clearly desirable. But it converges relatively slowly to that of the exact form, and thus the approximating order will be large if wideband accuracy is desired. By retaining the same idea of a passive representation, but enlarging the scope to include optimised designs, it is possible to obtain reasonable accuracy over a wide band of frequencies with approximations of a relatively low order, leading to much more efficient numerical methods. Accuracy in the very low frequency range is obviously not as good as for the continued fraction expansion, but can still be made very small, as illustrated in Figure 6. One weakness with the approach presented here is that, unlike the case of the continued fraction expansion, there is no longer a closed form expression for the element values, which must be reoptimised under changes in the temperature, tube radius, optimisation range or cost function - which is a trade-off typical of optimisation procedures in general.

\section{Acknowledgments}

This work was supported by the European Research Council, under grant number StG-2011-279068-NESS, as well as Labex MEC (ANR-10-LABX-0092) and the A*MIDEX project (ANR-11-IDEX-0001-02), funded by the Investissements d'Avenir program of the French National Research Agency (ANR).

${ }^{1}$ S. Thompson, T. Gabrielson, and D. Warren, "Analog model for thermoviscous propagation in a cylindrical tube", J. Acoust. Soc. Am. 135, 585-590 (2014).

2 J. Kergomard, "General equivalent electric circuits for acoustic horns", J. Audio Eng. Soc. 36, 948-955 (1988).

${ }^{3}$ S. Bilbao and J. Chick, "Finite difference time domain simulation for the brass instrument bore", J. Acoust. Soc. Am. 134, 3860-3871 (2013).

${ }^{4}$ D. Keefe, "Acoustical wave propagation in cylindrical ducts: Transmission line parameter approximations for isothermal and nonisothermal boundary conditions", Journal of the Acoustical Society of America 75, 58-62 (1984).

${ }^{5}$ L. Weinberg, Network Analysis and Synthesis, 312 (R. E. Kreiger, New York, New York) (1975).

6 A. Ben Jazia, B. Lombard, and C. Bellis, "Wave propagation in a fractional viscoelastic Andrade medium: Diffusive approximation and numerical modeling", Wave Motion 51, 994-1010 (2014).

7 T. Hélie and D. Matignon, "Diffusive representations for the analysis and simulation of flared acoustic pipes with visco-thermal losses", Math. Models Methods Appl. Sci. 16, 503-536 (2006). 